Acta Crystallographica Section E

Structure Reports

Online

ISSN 1600-5368

\section{Afroza Banu and G. M. Golzar Hossain*}

School of Chemistry, Cardiff University, Cardiff CF10 3AT, Wales

Correspondence e-mail: acsbd@yahoo.com

\author{
Key indicators \\ Single-crystal X-ray study \\ $T=120 \mathrm{~K}$ \\ Mean $\sigma(\mathrm{C}-\mathrm{C})=0.002 \AA$ \\ $R$ factor $=0.038$ \\ $w R$ factor $=0.105$ \\ Data-to-parameter ratio $=14.6$
}

For details of how these key indicators were automatically derived from the article, see http://journals.iucr.org/e.

\title{
Sulfathiazolium nitrate monohydrate
}

The title compound, $\mathrm{C}_{9} \mathrm{H}_{10} \mathrm{~N}_{3} \mathrm{O}_{2} \mathrm{~S}_{2}{ }^{+} \cdot \mathrm{NO}_{3}{ }^{-} \cdot \mathrm{H}_{2} \mathrm{O}$, was obtained from a solution of sulfathiazole in dilute nitric acid at room temperature. The crystal structure is stabilized by a network of hydrogen bonds and van der Waals interactions.

\section{Comment}

Sulfathiazole has a remarkable solvate-forming ability with interesting structural and conformational properties. Many solvent-containing sulfathiazoles are known and a lot of them have been studied crystallographically (Bingham et al., 2001).<smiles>CCCCCCC[NH2+]c1ccc(S(=O)(=O)N=c2[nH]ccs2)cc1</smiles>

(I)
Received 16 March 2006 Accepted 21 March 2006.

Shirotani et al. (1983) described three solvates of sulfathiazole and Caira et al. (1994) reported the crystal structure of the 1:1 complex of sulfathiazole and cyclodextrin, in which the molecules are hydrogen bonded with each other, forming a layer and these layers are linked by hydrogen bonds with water molecules.

The sulfathiazole molecule in the title complex, (I), is hydrogen bonded with a nitrate ion which is also hydrogen bonded with the water molecule. The sulfathiazole molecule is protonated on its terminal amino group.

The planes of the benzene and thiazole rings are inclined in a gauche conformation about the S12-N11 bond with a dihedral angle of $87.63(6)^{\circ}$. The crystal structure is stabilized by a network of hydrogen bonds and van der Waals interactions.

\section{Experimental}

Solid sulfathiazole $(0.255 \mathrm{~g} ; 1 \mathrm{mmol})$ was dissolved in $1 M \mathrm{HNO}_{3}$ acid $(50 \mathrm{ml})$ and stirred for 30 minutes, filtered off and the clear solution was left at room temperature for crystallization. Pale-yellow platelike crystals of sulfathiazole nitrate were obtained by slow evaporation of the solution.

\section{Crystal data}

$\mathrm{C}_{9} \mathrm{H}_{10} \mathrm{~N}_{3} \mathrm{O}_{2} \mathrm{~S}_{2}{ }^{+} \cdot \mathrm{NO}_{3}{ }^{-} \cdot \mathrm{H}_{2} \mathrm{O}$
$M_{r}=336.35$
Monoclinic, $P 2{ }_{1} / c$
$a=12.1917(2) \AA$
$b=7.6348(2) \AA$
$c=15.3895(2) \AA$
$\beta=107.4664(14)^{\circ}$
$V=1366.43(5) \AA^{3}$
$Z=4$

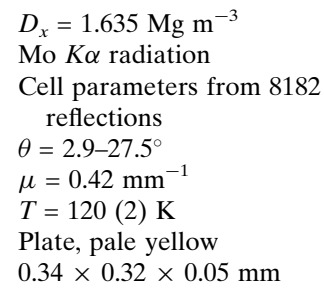




\section{Data collection}

Nonius KappaCCD diffractometer $\omega$ scans

Absorption correction: multi-scan (Blessing; 1995)

$T_{\min }=0.869, T_{\max }=0.979$

17799 measured reflections

3117 independent reflections

\section{Refinement}

Refinement on $F^{2}$

$R\left[F^{2}>2 \sigma\left(F^{2}\right)\right]=0.038$

$w R\left(F^{2}\right)=0.106$

$S=1.05$

3117 reflections

214 parameters

$\mathrm{H}$ atoms treated by a mixture of independent and constrained refinement

\section{Table 1}

Selected geometric parameters $\left(\AA{ }^{\circ}\right)$.

\begin{tabular}{llll}
\hline $\mathrm{S} 11-\mathrm{C} 11$ & $1.7362(17)$ & $\mathrm{N} 13-\mathrm{C} 17$ & $1.462(2)$ \\
$\mathrm{S} 11-\mathrm{C} 12$ & $1.732(2)$ & $\mathrm{C} 12-\mathrm{C} 13$ & $1.335(3)$ \\
$\mathrm{S} 12-\mathrm{O} 11$ & $1.4566(13)$ & $\mathrm{C} 14-\mathrm{C} 15$ & $1.381(2)$ \\
$\mathrm{S} 12-\mathrm{O} 12$ & $1.4436(13)$ & $\mathrm{C} 14-\mathrm{C} 19$ & $1.398(2)$ \\
$\mathrm{S} 12-\mathrm{N} 11$ & $1.5824(15)$ & $\mathrm{C} 15-\mathrm{C} 16$ & $1.392(2)$ \\
$\mathrm{S} 12-\mathrm{C} 14$ & $1.7740(17)$ & $\mathrm{C} 16-\mathrm{C} 17$ & $1.382(2)$ \\
$\mathrm{N} 11-\mathrm{C} 11$ & $1.344(2)$ & $\mathrm{C} 17-\mathrm{C} 18$ & $1.382(2)$ \\
$\mathrm{N} 12-\mathrm{C} 11$ & $1.335(2)$ & $\mathrm{C} 18-\mathrm{C} 19$ & $1.384(3)$ \\
$\mathrm{N} 12-\mathrm{C} 13$ & $1.384(2)$ & & \\
$\mathrm{C} 11-\mathrm{S} 11-\mathrm{C} 12$ & $90.84(8)$ & $\mathrm{C} 13-\mathrm{C} 12-\mathrm{S} 11$ & $111.26(14)$ \\
$\mathrm{O} 11-\mathrm{S} 12-\mathrm{O} 12$ & $117.06(8)$ & $\mathrm{C} 12-\mathrm{C} 13-\mathrm{N} 12$ & $112.90(17)$ \\
$\mathrm{O} 11-\mathrm{S} 12-\mathrm{N} 11$ & $104.67(8)$ & $\mathrm{C} 15-\mathrm{C} 14-\mathrm{C} 19$ & $121.33(15)$ \\
$\mathrm{O} 12-\mathrm{S} 12-\mathrm{N} 11$ & $114.68(8)$ & $\mathrm{C} 15-\mathrm{C} 14-\mathrm{S} 12$ & $120.22(13)$ \\
$\mathrm{O} 11-\mathrm{S} 12-\mathrm{C} 14$ & $106.67(8)$ & $\mathrm{C} 19-\mathrm{C} 14-\mathrm{S} 12$ & $118.45(13)$ \\
$\mathrm{O} 12-\mathrm{S} 12-\mathrm{C} 14$ & $106.87(8)$ & $\mathrm{C} 14-\mathrm{C} 15-\mathrm{C} 16$ & $119.52(15)$ \\
$\mathrm{N} 11-\mathrm{S} 12-\mathrm{C} 14$ & $106.17(8)$ & $\mathrm{C} 15-\mathrm{C} 16-\mathrm{C} 17$ & $118.86(15)$ \\
$\mathrm{C} 11-\mathrm{N} 11-\mathrm{S} 12$ & $120.76(13)$ & $\mathrm{C} 16-\mathrm{C} 17-\mathrm{C} 18$ & $121.95(16)$ \\
$\mathrm{C} 11-\mathrm{N} 12-\mathrm{C} 13$ & $115.14(15)$ & $\mathrm{C} 16-\mathrm{C} 17-\mathrm{N} 13$ & $119.15(15)$ \\
$\mathrm{N} 12-\mathrm{C} 11-\mathrm{N} 11$ & $119.41(15)$ & $\mathrm{C} 18-\mathrm{C} 17-\mathrm{N} 13$ & $118.90(15)$ \\
$\mathrm{N} 12-\mathrm{C} 11-\mathrm{S} 11$ & $109.85(12)$ & $\mathrm{C} 17-\mathrm{C} 18-\mathrm{C} 19$ & $119.43(16)$ \\
$\mathrm{N} 11-\mathrm{C} 11-\mathrm{S} 11$ & $130.74(14)$ & $\mathrm{C} 14-\mathrm{C} 19-\mathrm{C} 18$ & $118.90(16)$ \\
\hline
\end{tabular}

Table 2

Hydrogen-bond geometry $\left(\AA,^{\circ}\right)$.

\begin{tabular}{lllll}
\hline$D-\mathrm{H} \cdots A$ & $D-\mathrm{H}$ & $\mathrm{H} \cdots A$ & $D \cdots A$ & $D-\mathrm{H} \cdots A$ \\
\hline $\mathrm{N} 13-\mathrm{H} 13 A \cdots \mathrm{O} 1$ & $0.95(1)$ & $1.82(1)$ & $2.765(2)$ & $177(2)$ \\
$\mathrm{O} 4-\mathrm{H} 4 A \cdots \mathrm{O} 2$ & $0.95(1)$ & $1.87(1)$ & $2.8027(19)$ & $170(2)$ \\
$\mathrm{N} 12-\mathrm{H} 12 A \cdots \mathrm{O} 4{ }^{\mathrm{i}}$ & $0.95(1)$ & $2.00(1)$ & $2.9050(19)$ & $159(2)$ \\
$\mathrm{O} 4-\mathrm{H} 4 B \cdots \mathrm{O} 11^{\mathrm{i}}$ & $0.95(1)$ & $1.97(1)$ & $2.8807(18)$ & $162(2)$ \\
$\mathrm{N} 13-\mathrm{H} 13 C \cdots \mathrm{O} 11^{\mathrm{ii}}$ & $0.88(3)$ & $2.03(3)$ & $2.907(2)$ & $176(2)$ \\
$\mathrm{N} 13-\mathrm{H} 13 B \cdots \mathrm{O} 4{ }^{\mathrm{iii}}$ & $0.95(1)$ & $1.91(1)$ & $2.857(2)$ & $175(2)$ \\
\hline
\end{tabular}

Symmetry codes: (i) $-x+1, y+\frac{1}{2},-z+\frac{1}{2}$; (ii) $x,-y+\frac{1}{2}, z-\frac{1}{2}$; (iii) $-x+1,-y,-z$.

2669 reflections with $I>2 \sigma(I)$

$R_{\text {int }}=0.081$

$\theta_{\max }=27.5^{\circ}$

$h=-15 \rightarrow 15$

$=-9 \rightarrow 9$

$l=-19 \rightarrow 18$

$$
\begin{aligned}
& w=1 /\left[\sigma^{2}\left(F_{\mathrm{o}}{ }^{2}\right)+(0.0584 P)^{2}\right. \\
& +0.5659 P] \\
& \text { where } P=\left(F_{\mathrm{o}}{ }^{2}+2 F_{\mathrm{c}}{ }^{2}\right) / 3 \\
& (\Delta / \sigma)_{\max }=0.001 \\
& \Delta \rho_{\max }=0.47 \mathrm{e}^{-3} \\
& \Delta \rho_{\min }=-0.61 \mathrm{e}^{-3}
\end{aligned}
$$

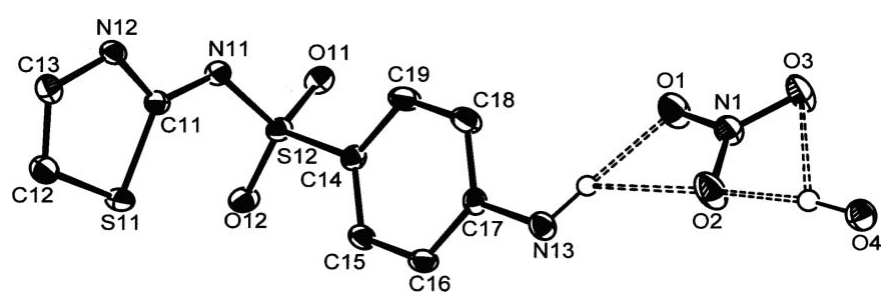

Figure 1

View of (I), showing the atom-labelling scheme. Displacement ellipsoids are drawn at the $50 \%$ probability level. $\mathrm{H}$ atoms not involved in hydrogen bonding (dashed lines) have been omitted.

C-bound $\mathrm{H}$ atoms were included in the riding model approximation with $\mathrm{C}-\mathrm{H}=0.95 \AA$, and with $U_{\text {iso }}(\mathrm{H})=1.2 U_{\text {eq }}(\mathrm{C})$. $\mathrm{H}$ atoms attached to $\mathrm{N}$ and $\mathrm{O}$ were located in an electron density map and refined isotropically with the $\mathrm{N}-\mathrm{H}$ and $\mathrm{O}-\mathrm{H}$ bond lengths restrained to 0.95 (5) $\AA$.

Data collection: COLLECT (Nonius, 1997-2000); cell refinement: HKL SCALEPACK (Otwinowski \& Minor 1997); data reduction: HKL DENZO (Otwinowski \& Minor 1997) and SCALEPACK; program(s) used to solve structure: SHELXS97 (Sheldrick, 1990); program(s) used to refine structure: SHELXL97 (Sheldrick, 1997); molecular graphics: ORTEP-3 for Windows (Farrugia, 1997); software used to prepare material for publication: Win $G X$ publication routines (Farrugia, 1999).

GMGH acknowledges the Ministry of Science and Technology, Bangladesh Secretariat, Dhaka for awarding the Bangabandhu Fellowship.

\section{References}

Bingham, A. L., Hughes, D. S., Hursthouse, M. B., Lancaster, R. W., Tavener, S. \& Threlfall, T. L. (2001). Chem. Commun. 7, 603-604.

Blessing, R. H. (1995). Acta Cryst. A51, 33-38.

Caira, M. R., Griffith, V. J., Nassimbeni, L. R., Luigi, R. \& Oudtshoom, B. V. (1994). J. Inclus. Phen. and Mol. Recog. in Chem. 17, 187-201.

Farrugia, L. J. (1997). J. Appl. Cryst. 30, 565.

Farrugia, L. J. (1999). J. Appl. Cryst. 32, 837-838.

Nonius (1997-2000). COLLECT. Nonius BV, Delft, The Netherlands.

Otwinowski, Z. \& Minor, W. (1997). Methods in Enzymology, Vol. 276, Macromolecular Crystallography, Part A, edited by C. W. Carter Jr and R. M. Sweet, pp. 307-326. New York: Academic Press.

Sheldrick, G. M. (1990). Acta Cryst. A46, 467-473.

Sheldrick, G. M. (1997). SHELXL97. University of Gottingen, Germany.

Shirotani, K.-I., Suzuki, E. \& Sekiguchi, K. (1983). Chem. Pharm. Bull. 31, 2085-2093. 


\title{
supporting information
}

Acta Cryst. (2006). E62, o1573-o1574 [https://doi.org/10.1107/S1600536806010312]

\section{Sulfathiazolium nitrate monohydrate}

\author{
Afroza Banu and G. M. Golzar Hossain
}

(I)

Crystal data

$\mathrm{C}_{9} \mathrm{H}_{10} \mathrm{~N}_{3} \mathrm{O}_{2} \mathrm{~S}_{2}^{+} \cdot \mathrm{NO}_{3}{ }^{-} \cdot \mathrm{H}_{2} \mathrm{O}$

$F(000)=696$

$M_{r}=336.35$

Monoclinic, $P 2_{1} / c$

Hall symbol: $-\mathrm{P} 2 \mathrm{ybc}$

$a=12.1917$ (2) $\AA$

$b=7.6348(2) \AA$

$c=15.3895(2) \AA$

$\beta=107.466(1)^{\circ}$

$V=1366.43(5) \AA^{3}$

$Z=4$

$D_{\mathrm{x}}=1.635 \mathrm{Mg} \mathrm{m}^{-3}$

Mo $K \alpha$ radiation, $\lambda=0.71073 \AA$

Cell parameters from 8182 reflections

$\theta=2.9-27.5^{\circ}$

$\mu=0.42 \mathrm{~mm}^{-1}$

$T=120 \mathrm{~K}$

Platelike, pale yellow

$0.34 \times 0.32 \times 0.05 \mathrm{~mm}$

\section{Data collection}

Nonius KappaCCD diffractometer

Radiation source: fine-focus sealed tube

17799 measured reflections

3117 independent reflections

Graphite monochromator

$\omega$ scans

Absorption correction: multi-scan

Blessing (1995)

$T_{\min }=0.869, T_{\max }=0.979$

2669 reflections with $I>2 \sigma(I)$

$R_{\text {int }}=0.081$

$\theta_{\max }=27.5^{\circ}, \theta_{\min }=3.0^{\circ}$

$h=-15 \rightarrow 15$

$k=-9 \rightarrow 9$

$l=-19 \rightarrow 18$

\section{Refinement}

Refinement on $F^{2}$

Least-squares matrix: full

$R\left[F^{2}>2 \sigma\left(F^{2}\right)\right]=0.038$

$w R\left(F^{2}\right)=0.106$

$S=1.05$

3117 reflections

214 parameters

5 restraints

Primary atom site location: structure-invariant direct methods

Secondary atom site location: difference Fourier map

Hydrogen site location: inferred from neighbouring sites

$\mathrm{H}$ atoms treated by a mixture of independent and constrained refinement

$w=1 /\left[\sigma^{2}\left(F_{\mathrm{o}}^{2}\right)+(0.0584 P)^{2}+0.5659 P\right]$ where $P=\left(F_{\mathrm{o}}^{2}+2 F_{\mathrm{c}}^{2}\right) / 3$

$(\Delta / \sigma)_{\max }=0.001$

$\Delta \rho_{\max }=0.47 \mathrm{e} \AA^{-3}$

$\Delta \rho_{\min }=-0.61$ e $\AA^{-3}$

\section{Special details}

Geometry. All e.s.d.'s (except the e.s.d. in the dihedral angle between two 1.s. planes) are estimated using the full covariance matrix. The cell e.s.d.'s are taken into account individually in the estimation of e.s.d.'s in distances, angles and torsion angles; correlations between e.s.d.'s in cell parameters are only used when they are defined by crystal symmetry. An approximate (isotropic) treatment of cell e.s.d.'s is used for estimating e.s.d.'s involving 1.s. planes. 
Refinement. Refinement of $F^{2}$ against ALL reflections. The weighted $R$-factor $w R$ and goodness of fit $S$ are based on $F^{2}$, conventional $R$-factors $R$ are based on $F$, with $F$ set to zero for negative $F^{2}$. The threshold expression of $F^{2}>\sigma\left(F^{2}\right)$ is used only for calculating $R$-factors(gt) etc. and is not relevant to the choice of reflections for refinement. $R$-factors based on $F^{2}$ are statistically about twice as large as those based on $F$, and $R$ - factors based on ALL data will be even larger.

Fractional atomic coordinates and isotropic or equivalent isotropic displacement parameters $\left(\hat{A}^{2}\right)$

\begin{tabular}{|c|c|c|c|c|}
\hline & $x$ & $y$ & $z$ & $U_{\text {iso }} * / U_{\text {eq }}$ \\
\hline S11 & $1.03911(4)$ & $0.33588(6)$ & $0.62604(3)$ & $0.02120(14)$ \\
\hline $\mathrm{S} 12$ & $0.80044(4)$ & $0.12718(6)$ & $0.62170(3)$ & $0.01730(13)$ \\
\hline O11 & $0.70722(11)$ & $0.07178(17)$ & $0.65647(8)$ & 0.0214 (3) \\
\hline $\mathrm{O} 12$ & 0.90388 (11) & 0.02267 (17) & $0.64704(8)$ & $0.0233(3)$ \\
\hline N11 & 0.81921 (13) & $0.32750(19)$ & $0.64842(10)$ & $0.0181(3)$ \\
\hline N12 & $0.91621(12)$ & $0.5878(2)$ & $0.64833(9)$ & $0.0172(3)$ \\
\hline $\mathrm{H} 12 \mathrm{~A}$ & $0.8512(12)$ & $0.648(2)$ & $0.6541(15)$ & $0.028(6)^{*}$ \\
\hline N13 & $0.61384(13)$ & $0.1154(2)$ & $0.21568(10)$ & $0.0190(3)$ \\
\hline H13A & $0.5326(5)$ & $0.121(3)$ & $0.2005(17)$ & $0.039(7)^{*}$ \\
\hline H13B & $0.6352(18)$ & $0.0138(17)$ & $0.1891(13)$ & $0.025(5)^{*}$ \\
\hline $\mathrm{H} 13 \mathrm{C}$ & $0.641(2)$ & $0.208(4)$ & 0.1947 (17) & $0.041(7)^{*}$ \\
\hline C11 & $0.91245(14)$ & $0.4134(2)$ & $0.64160(11)$ & $0.0168(3)$ \\
\hline $\mathrm{C} 12$ & $1.09071(15)$ & $0.5476(3)$ & $0.62748(12)$ & 0.0218 (4) \\
\hline $\mathrm{H} 12$ & 1.1631 & 0.5768 & 0.6201 & $0.026^{*}$ \\
\hline $\mathrm{C} 13$ & $1.01555(15)$ & $0.6644(2)$ & $0.64025(12)$ & 0.0203 (4) \\
\hline $\mathrm{H} 13$ & 1.0289 & 0.7871 & 0.6435 & $0.024 *$ \\
\hline $\mathrm{C} 14$ & $0.74583(14)$ & 0.1207 (2) & $0.50113(11)$ & 0.0165 (3) \\
\hline $\mathrm{C} 15$ & 0.80804 (14) & $0.0404(2)$ & $0.45070(12)$ & $0.0192(4)$ \\
\hline H15 & 0.8802 & -0.0122 & 0.4803 & $0.023^{*}$ \\
\hline $\mathrm{C} 16$ & $0.76426(14)$ & $0.0371(2)$ & $0.35615(12)$ & 0.0199 (4) \\
\hline H16 & 0.8058 & -0.0182 & 0.3205 & $0.024 *$ \\
\hline $\mathrm{C} 17$ & $0.65936(14)$ & $0.1156(2)$ & $0.31503(11)$ & $0.0158(3)$ \\
\hline $\mathrm{C} 18$ & $0.59642(15)$ & $0.1957(3)$ & $0.36506(12)$ & 0.0229 (4) \\
\hline H18 & 0.5243 & 0.2481 & 0.3351 & $0.027^{*}$ \\
\hline $\mathrm{C} 19$ & $0.63940(16)$ & $0.1989(3)$ & $0.45914(12)$ & $0.0240(4)$ \\
\hline H19 & 0.5973 & 0.2534 & 0.4946 & $0.029 *$ \\
\hline $\mathrm{O} 1$ & $0.37657(11)$ & $0.1342(2)$ & $0.16636(9)$ & $0.0291(3)$ \\
\hline $\mathrm{O} 2$ & $0.42291(11)$ & $0.1643(2)$ & $0.04127(9)$ & 0.0319 (4) \\
\hline $\mathrm{O} 3$ & $0.24360(11)$ & $0.15191(19)$ & $0.03616(9)$ & $0.0283(3)$ \\
\hline N1 & 0.34639 (13) & $0.1506(2)$ & $0.08093(10)$ & $0.0206(3)$ \\
\hline $\mathrm{O} 4$ & $0.31305(11)$ & $0.19590(17)$ & $-0.14618(9)$ & $0.0202(3)$ \\
\hline $\mathrm{H} 4 \mathrm{~A}$ & $0.346(2)$ & $0.198(3)$ & $-0.0823(4)$ & $0.047(7)^{*}$ \\
\hline $\mathrm{H} 4 \mathrm{~B}$ & $0.308(2)$ & $0.3159(10)$ & $-0.1620(16)$ & $0.039(7)^{*}$ \\
\hline
\end{tabular}

Atomic displacement parameters $\left(\AA^{2}\right)$

\begin{tabular}{lllllll}
\hline & $U^{11}$ & $U^{22}$ & $U^{33}$ & $U^{12}$ & $U^{13}$ & $U^{23}$ \\
\hline S11 & $0.0173(2)$ & $0.0231(2)$ & $0.0236(3)$ & $0.00481(17)$ & $0.00666(17)$ & $-0.00234(17)$ \\
S12 & $0.0199(2)$ & $0.0182(2)$ & $0.0132(2)$ & $0.00319(16)$ & $0.00403(16)$ & $-0.00013(15)$ \\
O11 & $0.0271(7)$ & $0.0204(6)$ & $0.0189(6)$ & $-0.0015(5)$ & $0.0104(5)$ & $0.0007(5)$
\end{tabular}




$\begin{array}{lllllll}\mathrm{O} 12 & 0.0258(7) & 0.0230(7) & 0.0178(6) & 0.0100(5) & 0.0018(5) & 0.0002(5) \\ \mathrm{N} 11 & 0.0179(7) & 0.0190(7) & 0.0171(7) & 0.0012(6) & 0.0048(5) & -0.0019(6) \\ \mathrm{N} 12 & 0.0143(7) & 0.0202(8) & 0.0161(7) & 0.0039(6) & 0.0029(5) & -0.0001(6) \\ \mathrm{N} 13 & 0.0157(7) & 0.0260(8) & 0.0149(7) & 0.0007(6) & 0.0038(6) & 0.0015(6) \\ \mathrm{C} 11 & 0.0152(8) & 0.0218(9) & 0.0115(8) & 0.0040(7) & 0.0013(6) & -0.0008(6) \\ \mathrm{C} 12 & 0.0177(8) & 0.0266(10) & 0.0216(9) & 0.0000(7) & 0.0064(7) & -0.0014(7) \\ \mathrm{C} 13 & 0.0164(8) & 0.0233(9) & 0.0201(9) & -0.0010(7) & 0.0036(7) & 0.0000(7) \\ \mathrm{C} 14 & 0.0185(8) & 0.0176(8) & 0.0128(8) & 0.0008(6) & 0.0037(6) & 0.0000(6) \\ \mathrm{C} 15 & 0.0150(8) & 0.0236(9) & 0.0176(8) & 0.0046(7) & 0.0027(6) & 0.0006(7) \\ \mathrm{C} 16 & 0.0181(8) & 0.0246(9) & 0.0183(8) & 0.0044(7) & 0.0077(6) & -0.0017(7) \\ \mathrm{C} 17 & 0.0152(8) & 0.0181(8) & 0.0133(8) & -0.0014(6) & 0.0030(6) & 0.0009(6) \\ \mathrm{C} 18 & 0.0167(8) & 0.0307(10) & 0.0207(9) & 0.0065(7) & 0.0049(7) & 0.0011(7) \\ \mathrm{C} 19 & 0.0205(9) & 0.0326(11) & 0.0198(9) & 0.0090(8) & 0.0073(7) & -0.0011(7) \\ \mathrm{O} 1 & 0.0212(7) & 0.0483(9) & 0.0166(7) & 0.0021(6) & 0.0041(5) & 0.0039(6) \\ \mathrm{O} 2 & 0.0167(6) & 0.0584(10) & 0.0217(7) & -0.0026(6) & 0.0075(5) & 0.0020(6) \\ \mathrm{O} 3 & 0.0135(6) & 0.0431(9) & 0.0252(7) & -0.0013(6) & 0.0010(5) & 0.0031(6) \\ \mathrm{N} 1 & 0.0174(7) & 0.0242(8) & 0.0201(8) & -0.0004(6) & 0.0053(6) & 0.0008(6) \\ \mathrm{O} 4 & 0.0189(6) & 0.0212(7) & 0.0205(6) & -0.0012(5) & 0.0061(5) & 0.0010(5)\end{array}$

Geometric parameters $\left(\AA,{ }^{\circ}\right)$

\begin{tabular}{llll}
\hline $\mathrm{S} 11-\mathrm{C} 11$ & $1.7362(17)$ & $\mathrm{C} 13-\mathrm{H} 13$ & 0.9500 \\
$\mathrm{~S} 11-\mathrm{C} 12$ & $1.732(2)$ & $\mathrm{C} 14-\mathrm{C} 15$ & $1.381(2)$ \\
$\mathrm{S} 12-\mathrm{O} 11$ & $1.4566(13)$ & $\mathrm{C} 14-\mathrm{C} 19$ & $1.398(2)$ \\
$\mathrm{S} 12-\mathrm{O} 12$ & $1.4436(13)$ & $\mathrm{C} 15-\mathrm{C} 16$ & $1.392(2)$ \\
$\mathrm{S} 12-\mathrm{N} 11$ & $1.5824(15)$ & $\mathrm{C} 15-\mathrm{H} 15$ & 0.9500 \\
$\mathrm{~S} 12-\mathrm{C} 14$ & $1.7740(17)$ & $\mathrm{C} 16-\mathrm{C} 17$ & $1.382(2)$ \\
$\mathrm{N} 11-\mathrm{C} 11$ & $1.344(2)$ & $\mathrm{C} 16-\mathrm{H} 16$ & 0.9500 \\
$\mathrm{~N} 12-\mathrm{C} 11$ & $1.335(2)$ & $\mathrm{C} 17-\mathrm{C} 18$ & $1.382(2)$ \\
$\mathrm{N} 12-\mathrm{C} 13$ & $1.384(2)$ & $\mathrm{C} 18-\mathrm{C} 19$ & $1.384(3)$ \\
$\mathrm{N} 12-\mathrm{H} 12 \mathrm{~A}$ & $0.945(5)$ & $\mathrm{C} 18-\mathrm{H} 18$ & 0.9500 \\
$\mathrm{~N} 13-\mathrm{C} 17$ & $1.462(2)$ & $\mathrm{C} 19-\mathrm{H} 19$ & 0.9500 \\
$\mathrm{~N} 13-\mathrm{H} 13 \mathrm{~A}$ & $0.948(5)$ & $\mathrm{N} 1-\mathrm{O} 1$ & $1.261(2)$ \\
$\mathrm{N} 13-\mathrm{H} 13 \mathrm{~B}$ & $0.949(5)$ & $\mathrm{N} 1-\mathrm{O} 2$ & $1.2632(19)$ \\
$\mathrm{N} 13-\mathrm{H} 13 \mathrm{C}$ & $0.88(3)$ & $\mathrm{N} 1-\mathrm{O} 3$ & $0.945(5)$ \\
$\mathrm{C} 12-\mathrm{C} 13$ & $1.335(3)$ & $\mathrm{O} 4-\mathrm{H} 4 \mathrm{~A}$ & $0.945(5)$ \\
$\mathrm{C} 12-\mathrm{H} 12$ & 0.9500 & $\mathrm{C} 12-\mathrm{C} 13-\mathrm{H} 13$ & 123.6 \\
$\mathrm{C} 11-\mathrm{S} 11-\mathrm{C} 12$ & & $\mathrm{~N} 12-\mathrm{C} 13-\mathrm{H} 13$ & 123.6 \\
$\mathrm{O} 11-\mathrm{S} 12-\mathrm{O} 12$ & $90.84(8)$ & $\mathrm{C} 15-\mathrm{C} 14-\mathrm{C} 19$ & $121.33(15)$ \\
$\mathrm{O} 11-\mathrm{S} 12-\mathrm{N} 11$ & $117.06(8)$ & $\mathrm{C} 15-\mathrm{C} 14-\mathrm{S} 12$ & $120.22(13)$ \\
$\mathrm{O} 12-\mathrm{S} 12-\mathrm{N} 11$ & $104.67(8)$ & $\mathrm{C} 19-\mathrm{C} 14-\mathrm{S} 12$ & $118.45(13)$ \\
$\mathrm{O} 11-\mathrm{C} 12-\mathrm{C} 15-\mathrm{C} 16$ & $119.52(15)$ \\
$\mathrm{O} 12-\mathrm{S} 12-\mathrm{C} 14$ & $114.68(8)$ & $\mathrm{C} 14-\mathrm{C} 15-\mathrm{H} 15$ & 120.2 \\
$\mathrm{~N} 11-\mathrm{S} 12-\mathrm{C} 14$ & $106.67(8)$ & $\mathrm{C} 16-\mathrm{C} 15-\mathrm{H} 15$ & 120.2 \\
$\mathrm{C} 11-\mathrm{N} 11-\mathrm{S} 12$ & $106.87(8)$ & $\mathrm{C} 15-\mathrm{C} 16-\mathrm{C} 17$ & $118.86(15)$ \\
$\mathrm{C} 11-\mathrm{N} 12-\mathrm{C} 13-\mathrm{H} 16$ & 120.6 \\
$\mathrm{C} 11-\mathrm{N} 12-\mathrm{H} 12 \mathrm{~A}$ & $106.17(8)$ & &
\end{tabular}




$\begin{array}{llll}\mathrm{C} 13-\mathrm{N} 12-\mathrm{H} 12 \mathrm{~A} & 125.7(13) & \mathrm{C} 15-\mathrm{C} 16-\mathrm{H} 16 & 120.6 \\ \mathrm{C} 17-\mathrm{N} 13-\mathrm{H} 13 \mathrm{~A} & 107.3(15) & \mathrm{C} 16-\mathrm{C} 17-\mathrm{C} 18 & 121.95(16) \\ \mathrm{C} 17-\mathrm{N} 13-\mathrm{H} 13 \mathrm{~B} & 112.6(13) & \mathrm{C} 16-\mathrm{C} 17-\mathrm{N} 13 & 119.15(15) \\ \mathrm{H} 13 \mathrm{~A}-\mathrm{N} 13-\mathrm{H} 13 \mathrm{~B} & 109(2) & \mathrm{C} 18-\mathrm{C} 17-\mathrm{N} 13 & 118.90(15) \\ \mathrm{C} 17-\mathrm{N} 13-\mathrm{H} 13 \mathrm{C} & 108.4(17) & \mathrm{C} 17-\mathrm{C} 18-\mathrm{C} 19 & 119.43(16) \\ \mathrm{H} 13 \mathrm{~A}-\mathrm{N} 13-\mathrm{H} 13 \mathrm{C} & 110(2) & \mathrm{C} 17-\mathrm{C} 18-\mathrm{H} 18 & 120.3 \\ \mathrm{H} 13 \mathrm{~B}-\mathrm{N} 13-\mathrm{H} 13 \mathrm{C} & 109(2) & \mathrm{C} 19-\mathrm{C} 18-\mathrm{H} 18 & 120.3 \\ \text { N12-C11-N11 } & 119.41(15) & \mathrm{C} 14-\mathrm{C} 19-\mathrm{C} 18 & 12.90(16) \\ \mathrm{N} 12-\mathrm{C} 11-\mathrm{S} 11 & 109.85(12) & \mathrm{C} 18-\mathrm{C} 19-\mathrm{H} 19 & 120.5 \\ \mathrm{~N} 11-\mathrm{C} 11-\mathrm{S} 11 & 130.74(14) & \mathrm{C} 14-\mathrm{C} 19-\mathrm{H} 19 & 119.04(15) \\ \mathrm{C} 13-\mathrm{C} 12-\mathrm{S} 11 & 111.26(14) & \mathrm{O} 1-\mathrm{N} 1-\mathrm{O} 2 & 120.80(15) \\ \mathrm{C} 13-\mathrm{C} 12-\mathrm{H} 12 & 124.4 & \mathrm{O} 1-\mathrm{N} 1-\mathrm{O} 3 & 120.16(15) \\ \mathrm{S} 11-\mathrm{C} 12-\mathrm{H} 12 & 124.4 & \mathrm{O} 2-\mathrm{N} 1-\mathrm{O} 3 & 103(2) \\ \mathrm{C} 12-\mathrm{C} 13-\mathrm{N} 12 & 112.90(17) & \mathrm{H} 4 \mathrm{~A}-\mathrm{O} 4-\mathrm{H} 4 \mathrm{~B} & \\ \end{array}$

Hydrogen-bond geometry $\left(A,{ }^{\circ}\right)$

\begin{tabular}{lllll}
\hline$D-\mathrm{H} \cdots A$ & $D-\mathrm{H}$ & $\mathrm{H} \cdots A$ & $D \cdots A$ & $D-\mathrm{H} \cdots A$ \\
\hline $\mathrm{N} 13-\mathrm{H} 13 A \cdots \mathrm{O} 1$ & $0.95(1)$ & $1.82(1)$ & $2.765(2)$ & $177(2)$ \\
$\mathrm{O} 4-\mathrm{H} 4 A \cdots \mathrm{O} 2$ & $0.95(1)$ & $1.87(1)$ & $2.8027(19)$ & $170(2)$ \\
$\mathrm{N} 12-\mathrm{H} 12 A \cdots \mathrm{O} 4^{\mathrm{i}}$ & $0.95(1)$ & $2.00(1)$ & $2.9050(19)$ & $159(2)$ \\
$\mathrm{O} 4-\mathrm{H} 4 B \cdots \mathrm{O} 11^{\mathrm{i}}$ & $0.95(1)$ & $1.97(1)$ & $2.8807(18)$ & $162(2)$ \\
$\mathrm{N} 13-\mathrm{H} 13 C \cdots \mathrm{O} 11^{\mathrm{ii}}$ & $0.88(3)$ & $2.03(3)$ & $2.907(2)$ & $176(2)$ \\
$\mathrm{N} 13-\mathrm{H} 13 B \cdots \mathrm{O} 44^{\mathrm{iii}}$ & $0.95(1)$ & $1.91(1)$ & $2.857(2)$ & $175(2)$
\end{tabular}

Symmetry codes: (i) $-x+1, y+1 / 2,-z+1 / 2$; (ii) $x,-y+1 / 2, z-1 / 2$; (iii) $-x+1,-y,-z$. 